8th Alexander Friedmann International Seminar

on Gravitation and Cosmology

International Journal of Modern Physics: Conference Series

Vol. 3 (2011) 87-97

(C) World Scientific Publishing Company

DOI: $10.1142 /$ S2010194511001176

\title{
WEYL GEOMETRY AS CHARACTERIZATION OF SPACE-TIME
}

\author{
F. P. POULIS and J. M. SALIM \\ Centro Brasileiro de Pesquisas Físicas, Rua Xavier Sigaud, 150 \\ Rio de Janeiro, RJ, CEP 22290-180, Brazil \\ fppoulis@cbpf.br,jsalim@cbpf.br \\ Received 14 June 2011 \\ Revised 11 July 2011
}

\begin{abstract}
Motivated by an axiomatic approach to characterize space-time it is investigated a reformulation of Einstein's gravity where the pseudo-riemannian geometry is substituted by a Weyl one. It is presented the main properties of the Weyl geometry and it is shown that it gives extra contributions to the trajectories of test particles, serving as one more motivation to study general relativity in Weyl geometry. It is introduced its variational formalism and it is established the coupling with other physical fields in such a way that the theory acquires a gauge symmetry for the geometrical fields. It is shown that this symmetry is still present for the red-shift and it is concluded that for cosmological models it opens the possibility that observations can be fully described by the new geometrical scalar field. It is concluded then that this reformulation, although representing a theoretical advance, still needs a complete description of their objects.
\end{abstract}

Keywords: Weyl geometry; gauge invariance; red-shift.

PACS numbers: 04.20.-q, 04.20.Cv, 04.90.+e

\section{Introduction}

General Relativity concerns a geometrical formulation of gravity and has as a tenet a pseudo-riemannian geometry for the manifolds taken in consideration. However, this is an arbitrary choice and is intended to preserve scalar products between vectors and, consequently, their lengths when parallel transported along an arbitrary path on the manifold.

Nevertheless, considering that in this theory the characterization of space-time is made by rulers and clocks based on light rays emission and reception, Ehlers, Pirani and Schild ${ }^{1}$ (EPS), followed by Woodhouse, ${ }^{2}$ have shown that the pseudoriemannian geometry represents a restriction among the possibilities those measuring tools allow. That is, starting from axioms concerning light rays ${ }^{\mathrm{a}}$ and trajectories of freely falling particles you get, necessarily, a Weyl geometry as the most general one. This, in turn, differs from the pseudo-riemannian one by allowing vectors to change their modulus when paralel transported along any curve.

${ }^{\text {a}}$ Described by a conformal invariant theory, like Maxwell's one. 
Weyl's motivation was to geometrize electromagnetism, with the intend to unify it with gravitation, and the length variation of vectors was associated with the electromagnetic potential. ${ }^{3}$ Being so, it suffered some criticisms that made it be abandoned. However, the axiomatic characterization of space-time by EPS and Woodhouse bring Weyl geometry to light once again. This time there is no association of length variation with any known physical field. The vector field that performs such a variation, which we will call $\omega_{\mu}$, is purely geometrical, and thus the objections raised before are transcended.

In Sec. 2 are presented the main properties of Weyl geometry. From them, preserving the postulate that test particles travel through time-like geodesics, it is verified that those should be different from the pseudo-riemannian case of General Relativity (GR). The $\omega_{\mu}$ field appears giving extra contributions to the trajectories of material particles and thus influencing on an observable of the theory. This is one of the motivations to study Einstein gravity in Weyl geometry: as a way to determine how or to what extent one can use the new geometric field to make the theory more consistent.

In the following section it is made a brief study of congruences of curves in Weyl. It is defined the concept of physical distance between neighboring curves and it is shown that their evolution is given by a linear transformation. Next, the matrix of this transformation is decomposed in their irreducible parts, which are the corresponding expressions for the expansion, shear and rotation from GR.

In Sec. 4 we restrict the Weyl geometry to its integrable version, where $\omega_{\mu} \equiv$ $\partial \omega / \partial x^{\mu}$. In this case, variations of length depend only on the starting and ending points of the trajectory. Variational formulations are presented for both obtaining gravitation in this geometry and for particles to follow geodesics. It is established how other physical fields are coupled with the new geometric one and it is observed that the coupling used endows our theory with a gauge symmetry inducing a conformal invariance.

As discussed in Ref. 4, since Dirac ${ }^{5}$ raised the question about the gravitational constant be, in fact, a constant, this problem has been a challenge for theoretical physics. However, it is argued that this can be solved by taking into account the principle that all fundamental equations of physics must be invariant under local transformations of scale, as proposed by Weyl ${ }^{3}$ and Hoyle and Narlikar. ${ }^{?}$ It is therefore of great theoretical interest to examine a conformal invariant version of gravitation.

Other versions of a non-conformal model for the gravitational interaction in Weyl Integrable Space-times (WIST) were already developed ${ }^{7,8}$ and applications were made in cosmology ${ }^{9,10}$ and in spherically symmetric configurations for modeling stars. ${ }^{11,12}$ Besides that, the WIST geometry has been used to describe the quantum behavior of a particle in an external potential in the non-relativistic ${ }^{13}$ and the relativistic ${ }^{14}$ regime. Nevertheless, none of them has formulated the theory in this gauge invariant way. 
Carrying on with the reformulation of the theory, it remained to redefine the observables of GR within this formalism. In Sec. 5 this is done for the red-shift and in Sec. 6 it is shown that it preserves the gauge symmetry of the theory, as expected. Moreover, in Sec. 7 it is shown that for cosmological models the conformal invariance opens the possibility that observations may no longer indicate a curvature nor an evolution to the metric. All observations and conclusions from the red-shift in GR being fully described in terms of the $\omega$ field.

We conclude that this reformulation of Einstein's gravitation in the more general and axiomatic context of Weyl geometries turns out to acquire a gauge symmetry that, although it represents a theoretical advance, introduces an indeterminacy of their objects which needs to be solved, for consistency.

\section{Some Properties of Weyl Geometry}

Weyl's geometry differs from that of Riemann by allowing vectors to also change their modules when parallel transported along a closed path. This can be summarized by the following expression for the covariant derivative of the metric: ${ }^{15}$

$$
\nabla_{\alpha} g_{\beta \gamma}=\omega_{\alpha} g_{\beta \gamma} \Leftrightarrow \nabla_{\alpha} g^{\beta \gamma}=-\omega_{\alpha} g^{\beta \gamma} .
$$

This implies a connection given by

$$
\Gamma_{\mu \nu}^{\alpha}=\hat{\Gamma}_{\mu \nu}^{\alpha}-\frac{1}{2}\left(\omega_{\mu} \delta_{\nu}^{\alpha}+\omega_{\nu} \delta_{\mu}^{\alpha}-g_{\mu \nu} \omega^{\alpha}\right)
$$

where symbols with hat refer to their equivalent in the Riemannian case.

From it, we can also conclude that for any vector $X^{\alpha}$ we have:

$$
\begin{aligned}
X_{\alpha} \nabla_{\beta} X^{\alpha} & =\frac{1}{2} \partial_{\beta}\left(X_{\alpha} X^{\alpha}\right)-\frac{1}{2} X^{\alpha} X_{\alpha} \omega_{\beta}, \\
X^{\alpha} \nabla_{\beta} X_{\alpha} & =\frac{1}{2} \partial_{\beta}\left(X_{\alpha} X^{\alpha}\right)+\frac{1}{2} X^{\alpha} X_{\alpha} \omega_{\beta} .
\end{aligned}
$$

Then, the most general geodesic equation is given by:

$$
u^{\beta} \nabla_{\beta} u^{\alpha}=\left(\frac{d}{d s} \ln \sqrt{u^{\beta} u_{\beta}}\right) u^{\alpha}-\frac{1}{2} u^{\beta} \omega_{\beta} u^{\alpha} .
$$

Where $u^{\alpha}$ is the tangent vector of the geodesic and $s$ is the parameter which describes it.

\section{Congruence of Curves}

We consider a congruence of curves $\gamma(\tilde{s}, t), C^{2}$, which in a local coordinate system $\left\{x^{\alpha}\right\}$, have coordinates $x^{\alpha}(\tilde{s}, t)$ and we take the parameter $\tilde{s}$ so that

$$
d \tilde{s}^{2}=e^{-\omega} d s^{2}=e^{-\omega} g_{\mu \nu} d x^{\mu} d x^{\nu}, \quad u^{\alpha}=\frac{d x^{\alpha}}{d \tilde{s}} \Rightarrow g_{\alpha \beta} u^{\alpha} u^{\beta}=e^{\omega} .
$$

Two neighboring points, $P\left(\tilde{s}_{0}, t_{0}\right) \in \gamma\left(\tilde{s}, t_{0}\right)$ and $Q\left(\tilde{s}_{0}, t_{0}+\Delta t\right) \in \gamma\left(\tilde{s}, t_{0}+\Delta t\right)$, define a new vector which we will call connection vector. Their components are 
$Z^{\alpha}=\partial x^{\alpha} /\left.\partial t\right|_{\substack{\tilde{s}=\tilde{s}_{0} \\ t=t_{0}}} \Delta t$ and it is associated with the distance, on the manifold, between the points $P$ and $Q$.

The physical distance, locally determined by an observer at rest in relation to what has the curve $\gamma\left(\tilde{s}, t_{0}\right)$ as world line, is given by $\perp Z^{\alpha} \equiv h_{\beta}^{\alpha} Z^{\beta}$, which we will call relative position vector, where $h_{\beta}^{\alpha}=\delta_{\beta}^{\alpha}-e^{-\omega} u^{\alpha} u_{\beta}$ makes the projection of any vector in the space perpendicular to $u^{\alpha}$.

Introducing the notation $\dot{X}^{\alpha} \equiv u^{\beta} \nabla_{\beta} X^{\alpha} \equiv \frac{D}{D \tilde{s}} X^{\alpha}$ to any vector $X^{\alpha}$, the relative velocity between two neighboring particles in the congruence is given by $\perp \frac{D}{D \widetilde{s}} \perp Z^{\alpha}$ and is related to $Z^{\alpha}$ by the following equation:

$$
\perp \frac{D}{D \tilde{s}} \perp Z^{\alpha}=h_{\beta}^{\alpha} u^{\gamma} \nabla_{\gamma}\left(h_{\delta}^{\beta} Z^{\delta}\right)=h_{\beta}^{\alpha} u^{\gamma} Z^{\delta} \nabla_{\gamma} h_{\delta}^{\beta}+h_{\delta}^{\alpha} \dot{Z}^{\delta} .
$$

By the property $\dot{Z}^{\delta}=u^{\alpha} \nabla_{\alpha} Z^{\delta}=Z^{\alpha} \nabla_{\alpha} u^{\delta}$, we can simplify the result to

$$
\begin{aligned}
\perp \frac{D}{D \tilde{s}} \perp Z^{\alpha} & =h_{\beta}^{\alpha} h_{\delta}^{\lambda}\left(\nabla_{\lambda} u^{\beta}\right) \perp Z^{\delta} \\
& =V^{\alpha}{ }_{\beta} \perp Z^{\beta}
\end{aligned}
$$

where we have defined $V^{\alpha}{ }_{\beta} \equiv h_{\gamma}^{\alpha} h_{\beta}^{\delta} \nabla_{\delta} u^{\gamma}$. This shows that the velocity of separation between neighboring particles is related to the relative position vector by a linear transformation.

It can be easily verified that $V_{\alpha \beta}=h_{\alpha}^{\lambda} h_{\beta}^{\delta} \nabla_{\delta} u_{\lambda}$, then we have their irreducible parts:

$$
\left.\begin{array}{l}
\theta_{\alpha \beta}=V_{(\alpha \beta)} ; \quad \theta=\theta_{\alpha}^{\alpha} \\
\sigma_{\alpha \beta}=\theta_{\alpha \beta}-\frac{1}{3} \theta h_{\alpha \beta} \\
\omega_{\alpha \beta}=V_{[\alpha \beta]}
\end{array}\right\} \Rightarrow V_{\alpha \beta}=\omega_{\alpha \beta}+\sigma_{\alpha \beta}+\frac{1}{3} \theta h_{\alpha \beta} .
$$

Where $V_{(\alpha \beta)}$ and $V_{[\alpha \beta]}$ are their symmetric and antisymmetric parts respectively. Although we are not with the riemannian connection anymore, the parameter we have defined is such that $\theta, \sigma_{\alpha \beta}$ and $\omega_{\alpha \beta}$ are still related to the expansion, shear and rotation of the congruence respectively. ${ }^{16}$

\section{Variational Principle and Conformal Invariant Theory}

In order to make this geometric description compatible with a variational principle for the dynamics of a particle in a gravitational field, ${ }^{17}$ we will restrict ourselves to a particular case of Weyl geometry which is called Weyl Integrable Space-time (WIST) in what follows. This is done simply by restricting the field $\omega_{\alpha}$ to a gradient. That is, from now on we will consider only

$$
\omega_{\alpha} \equiv \partial_{\alpha} \omega
$$

This kind of geometry is easily obtained by performing a Palatini approach to the action

$$
S=\int e^{-\omega} R \sqrt{-g} d^{4} x
$$


where $R$ is the Ricci scalar and we follow the sign conventions of Ref. 15. Variation of the connection gives precisely (2) with (10), what is necessary and suficient to give (1) ensuring we are in WIST. Variation of the metric and $\omega$ gives respectively:

$$
\begin{aligned}
& G_{\mu \nu}=0, \\
& R=0,
\end{aligned}
$$

where $G_{\mu \nu}$ is the Einstein tensor. We see that variation of $\omega$ gives a redundant equation, since (13) is the same as the trace of (12). Such equality implies in a freedom to one of the functions to be determined. Soon it will be shown what this freedom can imply in turn.

For test particles to obey the geodesic equation (5), we first note that in WIST we have

$$
u^{\beta} \omega_{\beta}=\frac{d x^{\beta}}{d s} \frac{\partial \omega}{\partial x^{\beta}}=\frac{d \omega}{d s}
$$

so, the equation becomes

$$
u^{\beta} \nabla_{\beta} u^{\alpha}=\left(\frac{d}{d s} \ln \sqrt{u^{\beta} u_{\beta}}\right) u^{\alpha}-\frac{1}{2} \frac{d \omega}{d s} u^{\alpha}=u^{\alpha} \frac{d}{d s} \ln \sqrt{e^{-\omega} g_{\mu \nu} u^{\mu} u^{\nu}} .
$$

Moreover, according to Refs. 18 and 19, in order to characterize a good clock for the observer who follows this geodesic, we should choose such a parameter in which the right hand side of the above equation is zero. This is achieved by precisely that same parameter $\tilde{s}$ defined before, in (6). This equation is obtained by the following action for a test particle with mass $m$ :

$$
S_{p}=\int 2 m \int_{s_{1}}^{s_{2}} \sqrt{e^{-\omega} g_{\mu \nu} \frac{d z^{\mu}}{d s} \frac{d z^{\nu}}{d s}} \delta^{4}(x-z(s)) d s d^{4} x .
$$

If we now notice that

$$
\begin{aligned}
& \Gamma_{\beta \gamma}^{\alpha}=\frac{1}{2} \tilde{g}^{\alpha \lambda}\left(\partial_{\beta} \tilde{g}_{\gamma \lambda}+\partial_{\gamma} \tilde{g}_{\beta \lambda}-\partial_{\lambda} \tilde{g}_{\beta \gamma}\right) \\
& \tilde{g}_{\mu \nu} \quad \equiv e^{-\omega} g_{\mu \nu}, \quad \tilde{g}^{\mu \nu} \equiv e^{\omega} g^{\mu \nu}
\end{aligned}
$$

that is, the connection is just like the Riemannian one (Christoffel symbol) written with $\tilde{g}_{\mu \nu}$, we see that things work much like they were in Riemann, but with $\tilde{g}_{\mu \nu}$ instead of $g_{\mu \nu}$ whenever it appears.

That is exactly what happened in the action (16) and hence in the geodesic equation (15). Specially if we take the parameter $s=\tilde{s}$ defined in Sec. 3, which is again another case of replacing $g_{\mu \nu}$ by $\tilde{g}_{\mu \nu}$ in the definition of the affine parameter in the riemannian case. Moreover, the situation is the same in (11): since we have $\sqrt{-\tilde{g}}=e^{-2 \omega} \sqrt{-g}$, the Einstein-Hilbert action becomes

$$
\begin{aligned}
S & =\int R\left(\tilde{g}^{\mu \nu}, \Gamma_{\beta \gamma}^{\alpha}\right) \sqrt{-\tilde{g}} d^{4} x=\int \tilde{g}^{\mu \nu} R_{\mu \nu}\left(\Gamma_{\beta \gamma}^{\alpha}\right) \sqrt{-\tilde{g}} d^{4} x= \\
& =\int e^{\omega} g^{\mu \nu} R_{\mu \nu}\left(\Gamma_{\beta \gamma}^{\alpha}\right) e^{-2 \omega} \sqrt{-g} d^{4} x=\int e^{-\omega} R\left(g^{\mu \nu}, \Gamma_{\beta \gamma}^{\alpha}\right) \sqrt{-g} d^{4} x .
\end{aligned}
$$


Now we have made these considerations, it is quite clear that the most natural choice for coupling other fields with $\omega$ is by keeping this same recipe. That is, we take any other lagrangian of the form $\mathcal{L}\left(g^{\mu \nu}, \ldots\right) \sqrt{-g}$, in GR, and write it as $\mathcal{L}\left(\tilde{g}^{\mu \nu}, \ldots\right) \sqrt{-\tilde{g}}$. Then, we will have for our WIST action:

$$
S=\int\left[R\left(\tilde{g}^{\mu \nu}, \Gamma_{\beta \gamma}^{\alpha}\right)+\mathcal{L}\left(\tilde{g}^{\mu \nu}, \ldots\right)\right] \sqrt{-\tilde{g}} d^{4} x .
$$

Variation of the connection gives us WIST. Variation with respect to the metric gives

$$
\begin{aligned}
& \frac{\delta S}{\delta g^{\mu \nu}}=\frac{\delta S}{\delta \tilde{g}^{\alpha \beta}} \frac{\delta \tilde{g}^{\alpha \beta}}{\delta g^{\mu \nu}}=\left[G_{\mu \nu}\left(\tilde{g}^{\mu \nu}\right)+T_{\mu \nu}\left(\tilde{g}^{\mu \nu}, \ldots\right)\right] e^{\omega} \sqrt{-\tilde{g}}=0 \Rightarrow \\
& \Rightarrow \quad G_{\mu \nu}\left(\tilde{g}^{\mu \nu}\right)=-T_{\mu \nu}\left(\tilde{g}^{\mu \nu}, \ldots\right), \quad R\left(\tilde{g}^{\mu \nu}\right)=T\left(\tilde{g}^{\mu \nu}, \ldots\right),
\end{aligned}
$$

where we have defined

$$
T_{\mu \nu}\left(\tilde{g}^{\mu \nu}, \ldots\right) \equiv \frac{1}{\sqrt{-\tilde{g}}} \frac{\delta\left[\mathcal{L}\left(\tilde{g}^{\mu \nu}, \ldots\right) \sqrt{-\tilde{g}}\right]}{\delta \tilde{g}^{\alpha \beta}} .
$$

Varying $\omega$ we have

$$
\begin{aligned}
& \frac{\delta S}{\delta \omega}=\frac{\delta S}{\delta \tilde{g}^{\mu \nu}} \frac{\delta \tilde{g}^{\mu \nu}}{\delta \omega}=\left[G_{\mu \nu}\left(\tilde{g}^{\mu \nu}\right)+T_{\mu \nu}\left(\tilde{g}^{\mu \nu}, \ldots\right)\right] e^{\omega} g^{\mu \nu} \sqrt{-\tilde{g}}=0 \Rightarrow \\
& \Rightarrow \quad R\left(\tilde{g}^{\mu \nu}\right)=T\left(\tilde{g}^{\mu \nu}, \ldots\right),
\end{aligned}
$$

which is a redundant equation, since it is equal to (21). So we still have a freedom to one of the functions to be determined. But now it is very clear where this freedom comes from. Since the function $\omega$ always appears together with the metric in the form $\tilde{g}_{\mu \nu}$, it is just a matter to see that it remains unchanged under the transformation

$$
\left\{\begin{aligned}
g_{\mu \nu} & \rightarrow \bar{g}_{\mu \nu}=e^{\Lambda} g_{\mu \nu} \\
\omega & \rightarrow \bar{\omega}=\omega+\Lambda
\end{aligned}\right.
$$

So we have an arbitrariness in the fields that keeps the theory just developed invariant.

Such invariance should be present in all observables defined on this theory, and so, our next task is to check it for the red-shift. We will now define it in this new context and show that it is invariant under the above transformation.

\section{Geometrical Optics and Red-Shift}

Following the lines of Ref. 20 we have for the geometrical optics approximation that light rays follow trajectories whose tangent vector, $k^{\alpha}$, is given by:

$$
k^{\alpha}=g^{\alpha \beta} k_{\beta}, \quad k_{\alpha} \equiv \partial_{\alpha} \varphi, \quad k^{\alpha} k_{\alpha}=0,
$$

where $\varphi$ is a phase. Moreover, according to (4), we also have $k^{\alpha} \nabla_{\beta} k_{\alpha}=0$. Since $\nabla_{\beta} k_{\alpha}=\nabla_{\alpha} k_{\beta}$, we then have $k^{\alpha} \nabla_{\alpha} k_{\beta}=0$. This, in turn, implies

$$
k^{\alpha} \nabla_{\alpha}\left(e^{\omega} k^{\beta}\right)=k^{\alpha} \hat{\nabla}_{\alpha} k^{\beta}=0 .
$$


That is, light rays follow null geodesics just like the manifold was riemannian instead of a Weyl one.

For the red-shift we have the following relation between the frequency of the electromagnetic waves in the moment of emission, $\nu_{e}$, and observation, $\nu_{o}$ :

$$
\frac{\nu_{e}}{\nu_{o}}=\frac{\left(k_{\alpha} u^{\alpha}\right)_{e}}{\left(k_{\beta} u^{\beta}\right)_{o}}
$$

The red-shift, $z$, of a source as measured by an observer is defined in terms of wavelenghts by

$$
z \equiv \frac{\lambda_{o}-\lambda_{e}}{\lambda_{e}} \equiv \frac{\Delta \lambda}{\lambda_{e}} \Rightarrow 1+z=\frac{\lambda_{o}}{\lambda_{e}}=\frac{\nu_{e}}{\nu_{o}}=\frac{\left(k_{\alpha} u^{\alpha}\right)_{e}}{\left(k_{\beta} u^{\beta}\right)_{o}},
$$

which determines the red-shift from the vectors $u_{e}^{\alpha}, u_{o}^{\alpha}$ and the tangent vector $k^{\alpha}$ of the null geodesic. This relation is valid regardless of the separation between the emitter and the observer and accounts for both Doppler and gravitational red-shift.

Next, we make the following decomposition of $k^{\alpha}$ : we consider an observer with four-velocity $u^{\alpha}$ and let $n^{\alpha}$ be a projection of $k^{\alpha}$ into the observer's rest frame, given by

$$
\begin{aligned}
n^{\alpha} & \equiv \frac{1}{u^{\gamma} k_{\gamma}} h_{\beta}^{\alpha} k^{\beta} \Rightarrow n^{\alpha} n_{\alpha}=-e^{-\omega}, n^{\alpha} u_{\alpha}=0, \\
k^{\alpha} & =u_{\beta} k^{\beta}\left(e^{-\omega} u^{\alpha}+n^{\alpha}\right) .
\end{aligned}
$$

Taking $v$ as the parameter along the null geodesic, we have $k^{\alpha}=d x^{\alpha} / d v$ and we can calculate the variation of $u^{\alpha} k_{\alpha}$ on an interval $d v$ along it as being ${ }^{\mathrm{b}}$

$$
d\left(u^{\alpha} k_{\alpha}\right)=\hat{\nabla}_{\beta}\left(u^{\alpha} k_{\alpha}\right) k^{\beta} d v=\left(\hat{\nabla}_{\beta} u_{\alpha}\right) k^{\alpha} k^{\beta} d v+u_{\alpha} \underbrace{k^{\beta} \hat{\nabla}_{\beta} k^{\alpha}}_{=0} d v .
$$

From (9) and (30) we can rewrite it as

$$
d\left(u^{\alpha} k_{\alpha}\right)=\left(\theta_{\alpha \beta} n^{\alpha} n^{\beta}+e^{-\omega} n^{\alpha} \dot{u}_{\alpha}\right)\left(u_{\gamma} k^{\gamma}\right)^{2} d v .
$$

Now, from (28) we have

$$
\frac{d \lambda}{\lambda}=-\frac{d\left(u^{\alpha} k_{\alpha}\right)}{\left(u^{\beta} k_{\beta}\right)}=-\left(\theta_{\alpha \beta} n^{\alpha} n^{\beta}+e^{-\omega} n^{\alpha} \dot{u}_{\alpha}\right) u_{\gamma} k^{\gamma} d v,
$$

which gives the variation of the wavelength along a small increment $d v$ on the parameter that describes the light ray.

Next, we show that both this expression and (28) are gauge invariants, as expected.

\footnotetext{
${ }^{\mathrm{b}}$ For the case where both emitter and observer follow the unique fluid velocity $u^{\alpha}$.
} 


\section{Verification of the Conformal Invariance of Red-Shift}

Let us examine the invariance of the objects that characterize the red-shift under the conformal transformation (24). We see that the following quantities from their definitions are transformed as follows:

$$
\begin{aligned}
& d \tilde{s}=e^{-\omega} g_{\mu \nu} d x^{\mu} d x^{\nu} \rightarrow d \tilde{s}, \\
& u^{\alpha} \equiv \frac{d x^{\alpha}}{d \tilde{s}} \rightarrow u^{\alpha}, \quad u_{\alpha}=g_{\alpha \beta} u^{\beta} \rightarrow e^{\Lambda} u_{\alpha}, \\
& k_{\alpha} \equiv \frac{\partial \varphi}{\partial x^{\alpha}} \rightarrow k_{\alpha}, \quad k^{\alpha}=g^{\alpha \beta} k_{\beta} \rightarrow e^{-\Lambda} k^{\alpha} .
\end{aligned}
$$

Thus, we have

$$
u^{\alpha} k_{\alpha} \rightarrow u^{\alpha} k_{\alpha}, \quad u_{\alpha} k^{\alpha} \rightarrow u_{\alpha} k^{\alpha},
$$

and we confirm the invariance of (28). For equation (33) we have

$$
\begin{aligned}
& h_{\beta}^{\alpha}=\delta_{\beta}^{\alpha}-e^{-\omega} u^{\alpha} u_{\beta} \rightarrow h_{\beta}^{\alpha}, \quad h_{\alpha \beta} \rightarrow e^{\Lambda} h_{\alpha \beta}, \quad h^{\alpha \beta} \rightarrow e^{-\Lambda} h^{\alpha \beta}, \\
& n^{\alpha}=\frac{1}{\left(u^{\gamma} k_{\gamma}\right)} h_{\beta}^{\alpha} k^{\beta} \rightarrow e^{-\Lambda} n^{\alpha}, \quad n_{\alpha} \rightarrow n_{\alpha},
\end{aligned}
$$

and their different terms are transformed as:

$$
\begin{aligned}
\theta_{\alpha \beta} n^{\alpha} n^{\beta}=n^{\alpha} n^{\beta} \nabla_{\alpha} u_{\beta} & \rightarrow e^{-2 \Lambda} n^{\alpha} n^{\beta} \nabla_{\alpha}\left(e^{\Lambda} u_{\beta}\right)=e^{-\Lambda} \theta_{\alpha \beta} n^{\alpha} n^{\beta} \\
\dot{u}_{\alpha} n^{\alpha} e^{-\omega} & \rightarrow e^{-\Lambda} \dot{u}_{\alpha} n^{\alpha} e^{-\omega}
\end{aligned}
$$

Given (37), it remains only to determine the transformation of $d v$, which is obtained from

$$
k^{\mu}=\frac{d x^{\mu}}{d v} \rightarrow e^{-\Lambda} k^{\mu} \quad \Leftrightarrow \quad d v \rightarrow e^{\Lambda} d v
$$

So we have

$$
\begin{aligned}
\frac{d \lambda}{\lambda}=-\left(\theta_{\alpha \beta} n^{\alpha} n^{\beta}+\right. & \left.e^{-\omega} n^{\alpha} \dot{u}_{\alpha}\right) u_{\gamma} k^{\gamma} d v \rightarrow \\
& \rightarrow-\left(e^{-\Lambda} \theta_{\alpha \beta} n^{\alpha} n^{\beta}+e^{-\Lambda} e^{-\omega} n^{\alpha} \dot{u}_{\alpha}\right) u_{\gamma} k^{\gamma} e^{\Lambda} d v=\frac{d \lambda}{\lambda},
\end{aligned}
$$

and we see that the gauge freedom of our theory still holds for the red-shift, preserving the arbitrariness to one of their geometrical objects.

\section{Consequences of Conformal Invariance in Cosmological Models}

We have the Friedmann model for the three possible curvatures in a Weyl geometry where, initially, $\omega=0$ :

$$
d s^{2}=d \tau^{2}-a^{2}(\tau)\left[d \rho^{2}+f^{2}(\rho) d \Omega^{2}\right],
$$

where $f(\rho)$ is equal to $\sin (\rho), \rho$ or $\sinh (\rho)$ for the spherical, flat and hyperbolic cases, respectively. The red-shift can be calculated from (27) or (33), where the velocities 
of the observer and emitter are both equal to

$$
u^{\alpha}=(1,0,0,0) .
$$

Now for $k^{\alpha}$, we have in the three models:

$$
\begin{aligned}
& k^{\alpha}=\frac{1}{a(\tau)}\left(1, \pm \frac{1}{a(\tau)}, 0,0\right) \quad \Leftrightarrow \quad k_{\alpha}=\frac{1}{a(\tau)}(1, \mp a(\tau), 0,0), \\
& k_{\alpha} u^{\alpha}=\frac{1}{a(\tau)} \Rightarrow 1+z=\frac{a\left(\tau_{o}\right)}{a\left(\tau_{e}\right)} .
\end{aligned}
$$

This expression can also be obtained from (33), since we have in it:

$$
\theta_{\alpha \beta} n^{\alpha} n^{\beta}=-\frac{1}{a(\tau)} \frac{d a(\tau)}{d \tau}, \quad \dot{u}_{\alpha}=0, \quad u_{\gamma} k^{\gamma} d v=u_{\gamma} d x^{\gamma}=d \tau,
$$

and the equation gets:

$$
\frac{d \lambda}{\lambda}=\frac{1}{a(\tau)} \frac{d a(\tau)}{d \tau} d \tau \Rightarrow d(\ln \lambda)=d(\ln a), \quad 1+z=\frac{\lambda_{o}}{\lambda_{e}}=\frac{a\left(\tau_{o}\right)}{a\left(\tau_{e}\right)} .
$$

However, being the metric (44) conformally flat, we can perform coordinate transformations in order to rewrite it as $d s^{2}=e^{-\Lambda} \eta_{\mu \nu} d x^{\mu} d x^{\nu}$ (see Appendix). Then, we make the transformation (24) and we are now with a Weyl geometry where $g_{\mu \nu}=\eta_{\mu \nu}, \omega=\Lambda$ and we have the same expression for the red-shift. That is, we now have the possibility that observations of red-shift can be attributed to the field $\omega$ of a Weyl geometry in Minkowski space. No longer indicating a curvature nor evolution to the metric. Likewise, we could have done something in between, i.e., a gauge transformation to get $\omega \neq 0$ and $g_{\mu \nu} \neq \eta_{\mu \nu}$.

\section{Conclusions}

Motivated by the axiomatic approach of EPS and Woodhouse to characterize spacetime we performed a reformulation of GR in the context of WIST for the sake of consistency.

After introducing the variational formulation of their basic concepts, like the dynamics for their geometrical objects and test particles, it was established how other physical fields couples with the geometry. The particular coupling choosen endows our reformulation with an unprecedented gauge symmetry that is of great theoretical interest by itself. On the other hand, this same gauge freedom leaves the role of their geometric fields undetermined. That is, one can choose any expression for one of them.

It was then presented a reformulation of the red-shift in which the symmetry is still present. It was observed that in the cosmological case the gauge freedom may be used to deprive the metric of any curvature or evolution. We can make, as a particular case, such a transformation in which the metric becomes the Minkowski one and the whole red-shift observations turns out to be described solely in terms of the new geometrical field, $\omega$. 
We see that, so far, this reformulation still lacks a complete description of their geometric objects. The attempt of having them fully described is our current subject of investigation.

\section{Acknowledgments}

We acknowledge Conselho Nacional de Desenvolvimento Científico e Tecnológico for financial support.

\section{Appendix A. Coordinate Transformations for the Cosmological Case}

We want to perform coordinate transformations in order to rewrite the metric (44) as

$$
d s^{2}=e^{-\Lambda}\left[d t^{2}-d r^{2}-r^{2} d \Omega^{2}\right] .
$$

Those are one for each case of the function $f(\rho)$, so we take each of them separatelly.

- Flat case:

$$
d s^{2}=d \tau^{2}-a^{2}(\tau)\left[d \rho^{2}+\rho^{2} d \Omega^{2}\right]=e^{-\Lambda(t)}\left[d t^{2}-d r^{2}-r^{2} d \Omega^{2}\right] .
$$

This is accomplished by making

$$
\begin{aligned}
& d t=\frac{d \tau}{a(\tau)}, \\
& r=\rho, \\
& e^{-\Lambda(t)}=A^{2}(t) \quad(A \equiv a \circ \tau) .
\end{aligned}
$$

- Hyperbolic case

$$
d s^{2}=d \tau^{2}-a^{2}(\tau)\left[d \rho^{2}+\sinh ^{2}(\rho) d \Omega^{2}\right]=e^{-\Lambda(\tau(t, r))}\left[d t^{2}-d r^{2}-r^{2} d \Omega^{2}\right] .
$$

This is achieved by the transformations:

$$
\left\{\begin{array} { l } 
{ r = e ^ { \frac { \Lambda ( \tau ) } { 2 } } a ( \tau ) \operatorname { s i n h } ( \rho ) , } \\
{ t = e ^ { \frac { \Lambda ( \tau ) } { 2 } } a ( \tau ) \operatorname { c o s h } ( \rho ) }
\end{array} \Leftrightarrow \left\{\begin{array}{l}
\rho=\tanh ^{-1}\left(\frac{r}{t}\right)=\frac{1}{2} \ln \left(\frac{t+r}{t-r}\right) \\
e^{\frac{\Lambda(\tau)}{2}} a(\tau)=\sqrt{t^{2}-r^{2}}
\end{array}\right.\right.
$$

With the condition that

$$
\frac{d \Lambda(\tau)}{d \tau}=-\frac{2}{a(\tau)} \frac{d}{d \tau}[a(\tau) \pm \tau] \quad \Leftrightarrow \quad \frac{d}{d \tau}\left[e^{\frac{\Lambda(\tau)}{2}} a(\tau)\right]=\mp e^{\frac{\Lambda(\tau)}{2}} .
$$

- Spherical case

$$
d s^{2}=d \tau^{2}-a^{2}(\tau)\left[d \rho^{2}+\sin ^{2}(\rho) d \Omega^{2}\right]=e^{-\Lambda(\tau(t, r))}\left[d t^{2}-d r^{2}-r^{2} d \Omega^{2}\right] .
$$

This is done by simply making the changes:

$$
\left\{\begin{array}{l}
a(\tau) \rightarrow \pm i a(\tau) \\
\rho \rightarrow \pm i \rho
\end{array}\right.
$$

in the transformation used for the hyperbolic case, where $i=\sqrt{-1}$. 
Given those transformations, it is straightforward to apply the gauge transformation (24) and check that everything actualy tranforms like it was shown in Sec. 6 . The final result (49) then follows necessarily.

\section{References}

1. J. Ehlers, F. A. E. Pirani and A. Schild, The geometry of free fall and light propagation, in General Relativity, Papers in Honour of J. L. Synge (Oxford U. P., Oxford, 1972).

2. N. M. J. Woodhouse, J. Math. Phys. 14, 495 (1973).

3. H. Weyl, Space, Time, Matter (Dover Publishers, New York, 1961).

4. J. D. Bekenstein and A. Meisels, Phys. Rev. D 22, 1313 (1980).

5. P. A. M. Dirac, Proc. Roy. Soc. London A 165, 199 (1938).

6. F. Hoyle and J. V. Narlikar, Action-at-a-Distance in Physics and Cosmology (Freeman, San Francisco, 1974); and in Cosmology, Fusion and Other Matters, ed. F. Reines, (Colorado Associated Universities Press, Boulder, 1972).

7. M. Novello, L. A. R. Oliveira, J. M. Salim and E. Elbaz, Int. J. Mod. Phys. D 1, 641 (1992).

8. J. M. Salim and S. L. Sautú, Class. Quant. Grav. 13, 353 (1996).

9. J. M. Salim, S. L. Sautú and H. P. de Oliveira, Class. Quant. Grav. 14, 2833 (1997).

10. J. M. Salim, J. C. Fabris and S. L. Sautú, Mod. Phys. Lett. A 13, 953 (1998).

11. J. M. Salim and S. L. Sautú, Class. Quant. Grav. 15, 203 (1998).

12. J. M. Salim and S. L. Sautú, Class. Quant. Grav. 16, 3281 (1999).

13. M. Novello, J. M. Salim and F. T. Falciano, Int. J. Geom. Meth. Mod. Phys. 8, 87 (2011).

14. F. T. Falciano, M. Novello and J. M. Salim, Found. Phys. 40, 1885 (2010).

15. R. Adler, M. Bazin and M. Schiffer, Introduction to General Relativity, 2nd edn. (McGraw-Hill, New York, 1975).

16. F. P. Poulis, PhD. thesis, in preparation.

17. J. Audretsch, Phys. Rev. D 27, 2872 (1983).

18. R. Chandra, Gen. Rel. Grav. 16, 1023 (1984).

19. V. Perlick, Gen. Rel. Grav. 19, 1059 (1987).

20. G. F. R. Ellis, Relativistic cosmology, in Proceedings of the International School of Physics "Enrico Fermi", Course 47: General Relativity and Cosmology, ed. R. K. Sachs (Academic Press, New York, 1971), p. 104; reprinted in Gen. Relativ. Gravit. 41, 581 (2009). 